\title{
Autonomy for underwater robots-a European perspective
}

\author{
Cécile Huet $^{1}$ - Franco Mastroddi ${ }^{1}$
}

Received: 20 August 2016 / Accepted: 25 August 2016 / Published online: 7 September 2016

(C) Springer Science+Business Media New York 2016

\begin{abstract}
The marine sector is one of the most promising development domains for the professional service robot sector. This article explores why autonomy is important and how it fits into the EU robotics programme. It looks at the big picture, encompassing research issues, as well as the industrial, socio-economic and regulatory landscape.
\end{abstract}

Keywords Autonomy · Long-term autonomy · Future perspective $\cdot$ European activities

\section{Introduction to the EU robotics programme}

Robots are expected to be more and more ubiquitous and to become a main contributor to the new digital economy. The European Commission's communication on digitising industry ${ }^{1}$ underlines the new opportunities provided by robotics, and is dedicated to make its citizens and its industry reap the benefits offered by such technology. The EU research and innovation programme in robotics supports this policy, through the development of smarter robots which are sufficiently flexible, robust and capable of interacting intuitively and safely with humans; the objective is to contribute to the growth of industry by improving their products, processes and services and to contribute to citizens' quality of life with robots fulfilling useful tasks, helping in their daily lives, at work and at home.

A bit of history: in 2004 the EU launched its "Cognitive Systems" programme and in 2006 its first call for proposals

Cécile Huet

cecile.huet@ec.europa.eu

1 Robotics and Artificial Intelligence, DG CONNECT, European Commission, Euroforum Building, 10, Rue Robert Stumper, Luxembourg L-2557, Luxembourg dedicated to advanced robotics. Today robotics in Horizon 2020, the EU's framework research programme is one of the largest civilian programmes of its kind worldwide. The main strategic aim is to boost Europe's industrial competitiveness through research, technological development, demonstration and innovation. ${ }^{2} \mathrm{H} 2020$ adds a clear industry and market dimension to the previous EU research programmes. It is aided in this by SPARC, ${ }^{3}$ the public-private partnership for robotics in Europe, which plays a key role here in getting the commitment of the robotics community, whether industry, academia or end-users. SPARC contributes directly to the programme through its Strategic Research Agenda for robotics in Europe.

Robotics is not only a sector in its own right but also transforms more traditional sectors such as manufacturing, automotive and other industry and service sectors. Robotics is essential for productivity and competitiveness through reindustrialisation and reshoring of manufacturing and production and stimulates new service markets ranging from autonomous cars to precision farming and smart homes. It also helps to address societal issues such as health and the ageing workforce. It has a growth potential especially in service markets such as healthcare, food production, environment, transport or security. The marine sector is particularly interesting for robotics, as it has a high socio-economic impact, especially in Europe, in terms of freight and passenger traffic, ${ }^{4}$ offshore industry and environmental protection needs.

\footnotetext{
${ }^{1}$ Digitising European Industry Reaping the full benefits of a Digital Single Market" (COM 2016) 180 final, 19/04/2016.

${ }^{2} \operatorname{COM}(2011) 811$ of 30.11 .2011$.

${ }^{3} \mathrm{http}: / /$ sparc-robotics.eu/.

${ }^{4}$ http://ec.europa.eu/eurostat/statistics-explained/index.php/ Maritime_ports_freight_and_passenger_statistics.
} 


\section{Importance of research into autonomy and potential for marine robotics}

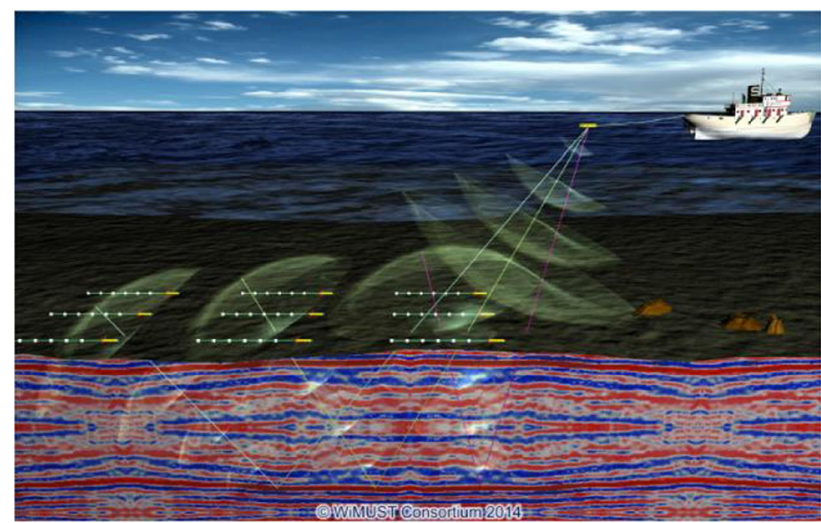

(Image courtesy of Wi-Must project)

The use of marine robots in industry, research and service applications has dramatically increased since the introduction of unmanned vehicles in the 1970s, but is still dominated by remotely operated vehicles (ROVs). The main technological barriers to wide deployment of autonomous underwater vehicles (AUVs) include:

- Low cost, easy to use and with persistent autonomy

- Autonomous obstacle avoidance

- Underwater communications

- Energy autonomy.

The research challenge in this domain is similar to many other fields of service robotics, however the highly dynamic characteristics and remoteness of the open sea magnifies some of these challenges greatly, for example through unpredictable currents, weather, obstacles and conditions which are generally hostile to normal human activity. Just staying in the same place is a major challenge for a hovering AUV, especially if it has to pick up an object on the seafloor or achieve precise manipulation tasks such as closing a pipe valve or picking up an artefact. Robustness is an absolute necessity, even for prototypes, at the low temperatures and high atmospheric pressures in the sea. Digital communications are greatly hindered underwater. Low light and marine snow impede computer vision constantly, unlike on land or in the air. Human-robot interaction underwater is on a smaller scale than on land, but is still very present in scenarios where a human operator is needed, for example research can address innovative interfaces such as haptics feedback for teleoperation or gesture recognition between AUV and diver. Autonomy is a research challenge which applies across the board; however an important point here is that full autonomy is not always possible or desired.

H2020 sets out key research priorities in the domain of Robotics and Autonomous Systems. The current H2020 work programme (2016-2017), targets key technical capabilities including: systems development; interaction; mechatronics; perception/navigation/cognition. It also targets system abilities: configurability; adaptability; dependability; motion capability; manipulation and grasping; perception; decisional autonomy and cognitive ability.

Most, if not all of these targets apply to the marine sector. For example, marine robots require a high level of decisional autonomy. AUVs need to be able to assess their own situation within the context of the operation and decide how to behave to ensure their safety while carrying out the mission objectives. Also, optimal use of usually finite robotic resources (sensors, actuators, energy supplies...) is a necessity.

\section{The SPARC multi-annual roadmap}

The SPARC PPP includes a marine topic group which has put forward its vision in the Multiannual roadmap of strengths, weaknesses, opportunities and barriers. ${ }^{5}$ It has identified the market for underwater systems as amongst the most valuable within professional service robots. There are 8300 ports worldwide, over 620 mobile offshore drilling rigs (2010 figures) some of which cost several billion euros. Over the period 2006-2010 the average annual world demand for marine supplies was in the range of 149 billion EUR, fed by tens of thousands of companies in Europe alone, with hundreds of thousands of employees (Competitive Position and Future Opportunities of the European Marine Supplies Industry, European Commission, 2014). Environmental aspects also are an important issue. Pollution monitoring in ports is very costly. According to IUCN, the International Union for Conservation of Nature (2015) there are close to 5000 Marine Protected Areas representing 2.85 million $\mathrm{km}^{2}$.

Europe, with its extensive sea borders, has a highly developed marine and offshore industry. Yet as elsewhere in the world, the main barrier to opening the market of marine robots in daily civilian coastal and harbour applications is created by the lack of rules on the operations of unmanned vehicles at sea and the consequent legal issues, in particular concerning liability in the case of accident.

\section{Regulatory implications of autonomy}

In general, the regulatory landscape for robotics in Europe is fragmentary. This is not only because national legal systems differ. Robotics relates to many different regulation domains, ranging from liability and machine safety to data privacy.

\footnotetext{
5 http://sparc-robotics.eu/wp-content/uploads/2014/05/H2020Robotics-Multi-Annual-Roadmap-ICT-2016.pdf.
} 
Liability is a key issue. Who is to blame if an AUV injures someone? There is some progress in other fields, notably driverless cars, where Volvo says ${ }^{6}$ it will accept full liability for accidents involving its driverless cars, making it one of the first car companies to do so.

Safety regulations abound in the maritime sector, principally through major international shipping conventions, adopted by the International Maritime Organization (and the International Labour Organization) concerning safety and pollution prevention. Many other maritime instruments concerning more specific issues are also in force worldwide. One example is COLREG (Convention on the International Regulations for Preventing Collisions at Sea, 1972) which lays down the basic "rules of the road", such as rights of way and actions to avoid collisions. Research can help here, through the design and development of reliable autonomous anti-collision systems.

The explosion in sensor data has recently raised questions about privacy and data protection. Aerial drones are a case in point. For AUVs, this would probably not apply on a similar potentially large scale. But the question of who keeps, or has access to sensor data from the open sea which AUVs capture-for scientific, public or for commercial reasons-is still open. Protection of data generated by autonomous systems also affects the digital single market in Europe. This is relevant for industry as well as users. Clear rules are needed on the ownership and exploitation of data collected and used by any system, autonomous or not.

\section{EU-funded projects}

The EU currently funds a total of over 100 projects with 700 research partners around Europe. Most projects address the 'cognitive' cycle of perception-understanding-action and how to integrate new components, tools and methods into this cycle. Projects address many different application areas (e.g. industry and manufacturing, professional and domestic, medical and rehabilitation) and domains (e.g. aerial, land and marine).

The key common technological aim is to develop artificial systems that operate in dynamic real-life environments.

The marine world reflects this kind of environment par excellence. It poses many scientific and technical problems which have more in common with outer space than with land or aerial environments on Earth: remoteness, poor communications, high atmospheric pressure, low temperatures, lack of light and so on. On the other hand the marine domain is very important as mentioned above.

EU projects in the marine sector address such problems by developing new methods for sensing and perception, navigation and mapping, cooperation in teams, and intervention and manipulation. Methods are sometimes bio-inspired, and are driven by different applications needs such as monitoring pollution, harbour patrolling for ship guidance or security. The table below gives a sample list of such projects.

In addition to projects, the EU programme supports robotic competitions, focussing on demonstrating scientific and technical progress. The euRathlon 2014 marine robotics competition was held at the NATO Centre for Maritime Research and Experimentation in La Spezia, Italy. Competitors had to deal with real life conditions (i.e. limited visibility and salty water). 6 teams from 4 different countries participated in the competition. Scenarios faced by the robots included long range autonomous underwater navigation, underwater leak localization and structure inspection, or environmental survey of an accident area. ${ }^{7}$ The EU also supports training and exchanges in the marine sector e.g. through projects like Robocademy and Excellabust. ${ }^{8}$

$\overline{6}$ http://www.bbc.com/news/technology-34475031.

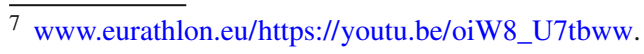

${ }^{8}$ http://www.robocademy.eu/http://excellabust.fer.hr/. 


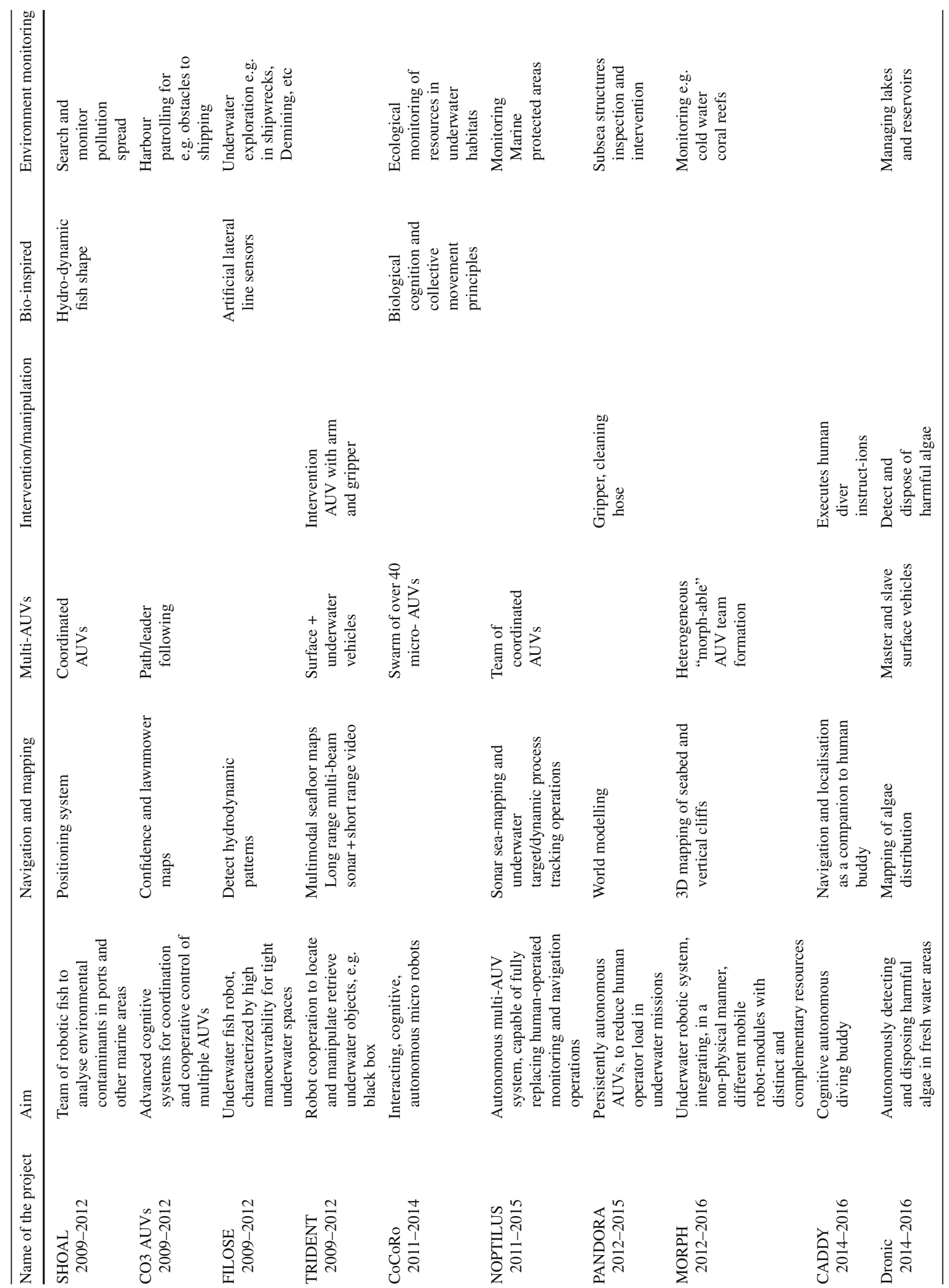




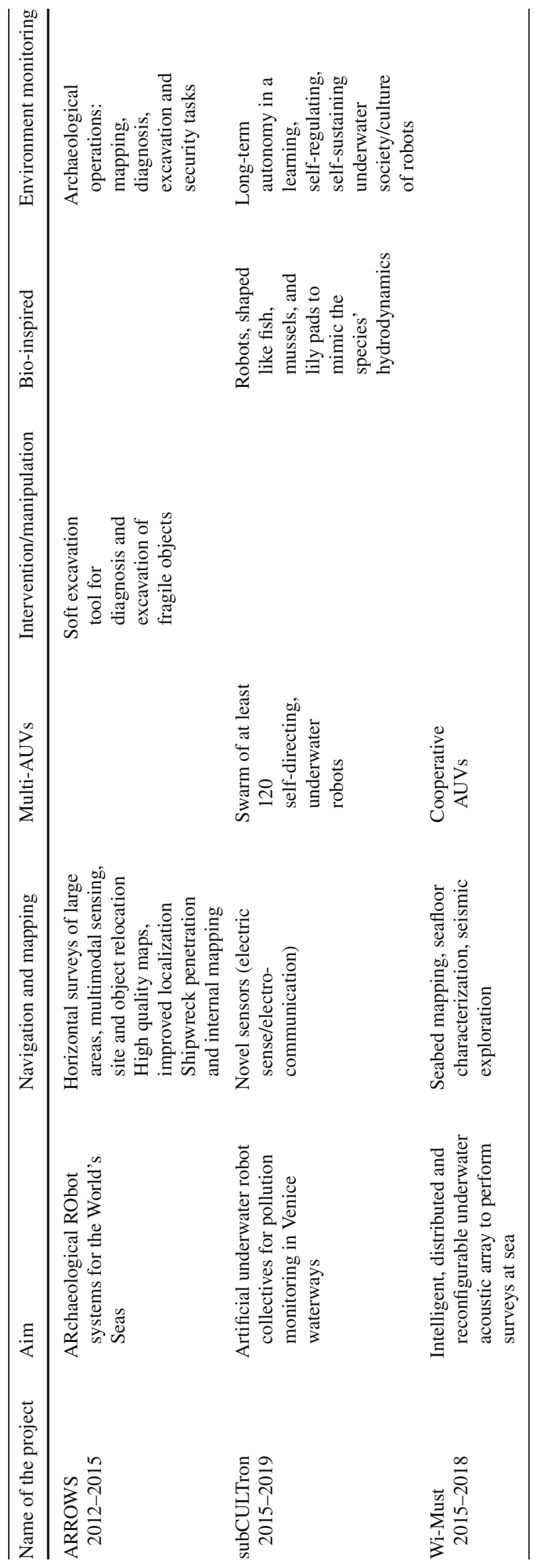

\section{Conclusions and way forward}

The $\mathrm{H} 2020$ robotics programme is expected to continue into and beyond 2020, adapting to major technological and socio-economic challenges along the way. Progress in digital technologies is expected to continue, changing the way we design, produce, commercialise and generate value from products and related services. Advances in technologies such as robotics are transforming products, processes and business models in all sectors, ultimately creating new industrial patterns as global value chains shift. The challenge ahead is for industry to seize fully and swiftly these digital opportunities.

Marine robotics will hopefully continue to play a strong role here, not only in the form of future projects but also through initiatives to create new competence centres or Digital Innovation Hubs. ${ }^{9}$ Such hubs in the marine robotics context, would provide a shared infrastructure, offering access to real-world testing facilities to the entire community, from academia, to industry, to potential users and could make a real contribution to advancing the technology and support technology transfer; they could, provide and exploit not only shared technical infrastructure, facilities and training support but also provide brokerage between users, suppliers and investors. Such Hubs can demonstrate what marine robotics can achieve and problems it can solve. They can lend engineering support for the integration of research results, help to compare approaches and push the limits of the technology, foster innovation and take up through new eco-systems. They can also provide an avenue to combine EU-level research with other programmes at regional and national level.

Such Hubs need not necessarily be developed from scratch but can build on existing infrastructures, of which there are several examples in Europe. For example, the Nato-Centre for Maritime Research and Experimentation (CMRE), IST Lisbon at the Expo 98 test site, Heriot Watt University at Fort William, Scotland, Girona test facilities etc.

With specific regard to autonomy, new research avenues are opening, for example based on recent progress in the field of Artificial Intelligence systems. AI methods increase the autonomous capabilities of robots through reasoning, planning, (deep) learning, natural language processing, enhanced perception, locomotion and manipulation. In the case of marine robotics, we have already seen an AI planner developed in the PANDORA project, for dealing with scheduled and unplanned events during a mission. AI also holds a prospect of exploiting massive troves of data for learning

\footnotetext{
${ }^{9}$ Commissioner Oettinger: "My objective is to have at least one world class digital innovation hub in every region in Europe" https://ec .europa.eu/commission/2014-2019/oettinger/announcements/speech-h annover-messe-europes-future-digital_en.
} 
about the environment and for combination with real time sensor data. Advances in AI brings a new dimension to robotics, not only reinforcing research in this field, but also allowing to develop a bridge with other fields, such as the Internet of Things or Big Data.

In conclusion, long-term autonomy is here for the long term.

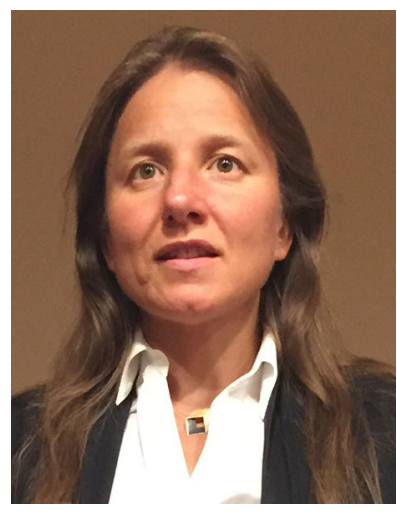

Cécile Huet is Deputy Head of the Unit "Robotics and Artificial Intelligence" in charge of the robotics programme at the European Commission.

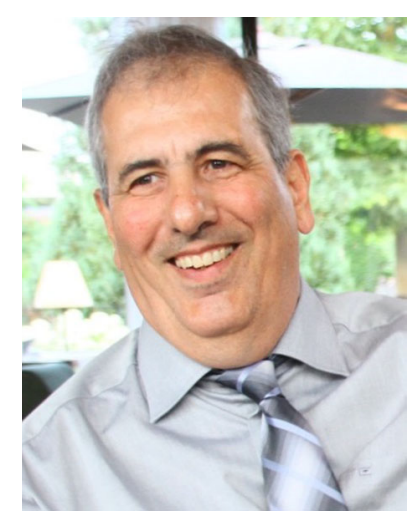

Franco Mastroddi is a programme officer with long experience of EU research in the robotics and cognitive systems domain. 